Draft Version OCtober 22, 2018

Preprint typeset using $\mathrm{L}^{A} \mathrm{~T}_{\mathrm{E}} \mathrm{X}$ style emulateapj v. 11/10/09

\title{
DERIVING THE GLOBAL STRUCTURE OF THE GALACTIC MAGNETIC FIELD FROM FARADAY ROTATION MEASURES OF EXTRAGALACTIC SOURCES
}

\author{
M. S. Pshirkov ${ }^{1,2}$, P.G.Tinyakov ${ }^{1,3}$, P.P.Kronberg ${ }^{4,5,6}$ and K. J. Newton-McGee ${ }^{6,7}$ \\ ${ }^{1}$ Universite Libre de Bruxelles, Service de Physique Theorique, CP225, 1050, Brussels, Belgium; pshirkov@ulb.ac.be \\ ${ }^{2}$ Pushchino Radio Astronomy Observatory, 142290, Pushchino, Russia \\ ${ }^{3}$ Institute for Nuclear Research, 117312, Moscow, Russia \\ ${ }^{4}$ Department of Physics, University of Toronto, Toronto M5S 1A7, Canada \\ 5 IGPP, Los Alamos National Laboratory, M.S. T006, Los Alamos NM 87545, USA \\ 6 Sydney Institute for Astronomy, School of Physics, The University of Sydney, NSW 2006, Australia \\ 7 Australia Telescope National Facility, CSIRO, PO Box 76, Epping NSW 1710, Australia \\ Draft version October 22, 2018
}

\begin{abstract}
We made use of the two latest sets of rotational measures (RMs) of extra-galactic radio sources, namely the NRAO VLA Sky Survey rotation measures catalog, and a compilation by Kronberg and Newton-McGee, to infer the global structure of the Galactic magnetic field (GMF). We have checked that these two data sets are mutually consistent. Given the existence of clear patterns in all-sky RM distribution we considered GMF models consisting of two components: disk (spiral or ring) and halo. The parameters of these components were determined by fitting different model field geometries to the observed RMs. We found that the model consisting of a symmetric (with respect to the Galactic plane) spiral disk and anti-symmetric halo fits the data best and reproduces the observed distribution of RMs over the sky very well. We confirm that ring disk models are disfavored. Our results favor small pitch angles around $\sim-5^{\circ}$ and an increased vertical scale of electron distribution, in agreement with some recent studies. Based on our fits, we select two benchmark models suitable for studies of cosmic ray propagation, including the ultra-high energies.
\end{abstract}

Subject headings: Galaxy: structure -ISM: magnetic fields- methods: data analysis

\section{INTRODUCTION}

A realistic model of the Galactic magnetic field (GMF) is needed for various applications, such as direct dark matter searches, studies of cosmic rays, and others. In particular, the GMF plays a crucial role in the propagation of ultra-high energy cosmic rays (UHECRs) - those with energies in excess of $10^{19} \mathrm{eV}$. Their deflections (assuming these are charged particles) in the GMF are large enough to prevent the identification of some, perhaps most sources directly from the UHECR data. However, in the case of protons these deflections are sufficiently small to be corrected for, provided the GMF is known with enough accuracy. Thus, the possibility of astronomy with charged particles at ultra-high energies may depend crucially on our knowledge of the GMF.

The magnetic field (MF) of our Galaxy is thought to contain both regular and turbulent components (see, e.g., Beck (2001, 2008)). While the regular component is subdominant in strength by a factor of a few, it is expected to give a dominant contribution in integral quantities such as total deflections of ultra-high energy particles. Unlike the turbulent component, the regular component adds up coherently and comes to dominate (Tinvakov \& Tkachev 2005) when integrated over many coherence lengths. Estimating the strength and global structure of the regular MF of our Galaxy is the main purpose of this paper. Our primary interest is on MF outside the thin Galactic disk, since that is the field that determines the deflections for most arrival directions of UHECRs.

The regular component of the GMF has been studied previously e.g. (Simard-Normandin \& Kronberg 1980; Sofue \& Fujimoto 1983; Han \& Qiao 1994; Frick et al.
2001; Vallée 2005; Han et al. 2006; Brown et al. 2007; Sun et al. 2008; Jansson et al. 2009), and in particular in the context of UHECR propagation (Stanev 1997; Tinyakov \& Tkachev 2002; Prouza \& Smída 2003). The advance of this paper is that it is based on improved data on rotation measures (RMs) and the ionized gas distribution. We also develop improved numerical model commensurate with this improved data.

Observations of other spiral galaxies reveal MFs of a few $\mu \mathrm{G}$ strength, coherent over distances of several kiloparsecs (see, e.g., Beck 2008; Krause 2009; Braun et al. 2010)). Observations from the face-on and edge-on perspectives indicate that these fields consist of at least two components: the 'disk' one which is concentrated towards the galactic plane and has largely a spiral pattern, and the 'halo' one that resides at some distance from the disk plane and has radial size comparable with the size of the galaxy. As the Milky Way seems to be a typical member of the entire class of spiral galaxies, MFs of similar strength and configuration should be expected in our Galaxy.

There are several methods to estimate the MF in the Galaxy, such as Zeeman splitting measurements (Crutcher 1999), infrared polarization studies (Nishiyama et al. 2010), synchrotron polarization and intensity surveys (Wielebinski 2005; Sun et al. 2008; Jansson et al. 2009; Jaffe et al. 2010; Ruiz-Granados et al. 2010), starlight polarization studies (Heiles 1996) and, finally, observation of the Faraday RMs of different Galactic (pulsars) and extragalactic radio sources (e.g.r.s.) (Simard-Normandin et al. 1981; Han et al. 2006; Men et al. 2008; Mao et al. 2010; Kronberg \& Newton-McGee 2011). Except for the 
Zeeman measurements all these methods are somewhat indirect as they involve additional assumptions such as density of warm ionized medium or concentrations of cosmic ray electrons. In this paper we use recent data on the Faraday RMs.

When propagating through a magnetized plasma, the polarization plane of a linearly polarized electromagnetic wave of wavelength $\lambda$ rotates by the angle $\Delta \psi$ proportional to the square of the wavelength,

$$
\Delta \psi=\mathrm{RM} \cdot \lambda^{2} .
$$

Multi-frequency observations thus allow one to measure the value of RM. The RM can be expressed in terms of the properties of the intergalactic/interstellar medium (IGM/ISM) and permeating MFs:

$$
\mathrm{RM}=0.81 \int_{0}^{\mathrm{D}} \mathrm{n}_{\mathrm{e}} \mathrm{B}_{||} \mathrm{dl},
$$

where $n_{e}$ is the density of free electrons measured in $\mathrm{cm}^{-3}, B_{\|}$is the component of the MF parallel to the line of sight measured in $\mu \mathrm{G}$ (positive when directed towards the observer), and $D$ is the distance from the observer to the source in pc. Combined with data on the Galactic free electron distribution, Equations (1) and (2) can be used to estimate the GMF from the Faraday RMs of a large number of sources.

Two types of sources are normally used to measure Galactic Faraday rotation: Galactic pulsars and extragalactic radio sources. Pulsars have an advantage that their intrinsic RM values are negligibly small. However, the use of pulsars requires the knowledge of their distances (usually inferred from their dispersion measures DM), which introduces additional assumptions about the ISM density distribution. In addition, pulsars are concentrated around the Galactic plane, and their total number $(\sim 500)$ is currently much smaller than the number of extragalactic radio sources with measured RMs. For these reasons in this paper, we concentrate on the RMs of the extragalactic radio sources.

Our general strategy is the following. We consider several types of analytical GMF models, each containing a finite number of parameters. For each model we calculate the expected RMs, and fit them to the observations by the binned $\chi^{2}$ method. The quality of the fit is then used to determine the best values of the GMF parameters and their allowed ranges.

The paper is organized as follows. Section 2 describes the data. Sections 3 and 4 describe the implemented models and our method. The results are presented in Section 5. Section 6] contains the results and conclusions.

\section{DATA}

In the present work we use two sets of the RM data. The first one is the compilation of RMs recently obtained by Taylor et al. (2009) who reanalyzed NRAO VLA Sky Survey (NVSS) data. NVSS is the largest by number survey of polarized radio sources at declinations $>-40^{\circ}$ (Condon et al. 1998). It was made in two nearby bands, 1364.9 and $1435.1 \mathrm{MHz}$; each having a width of $42 \mathrm{MHz}$. Simultaneous observations in these two different bands give estimates of the RMs of the sources. The total number of the observed sources was 37,543 with the mean error, estimated by the authors, of $\sim 11 \mathrm{rad} \mathrm{m}^{-2}$. The

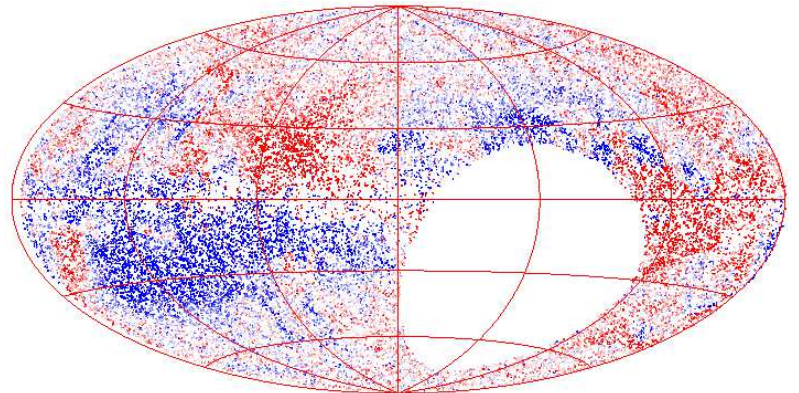

FIG. 1. - Locations and RM signs of the NVSS sources. Red (blue) color corresponds to positive (negative) values of RM.
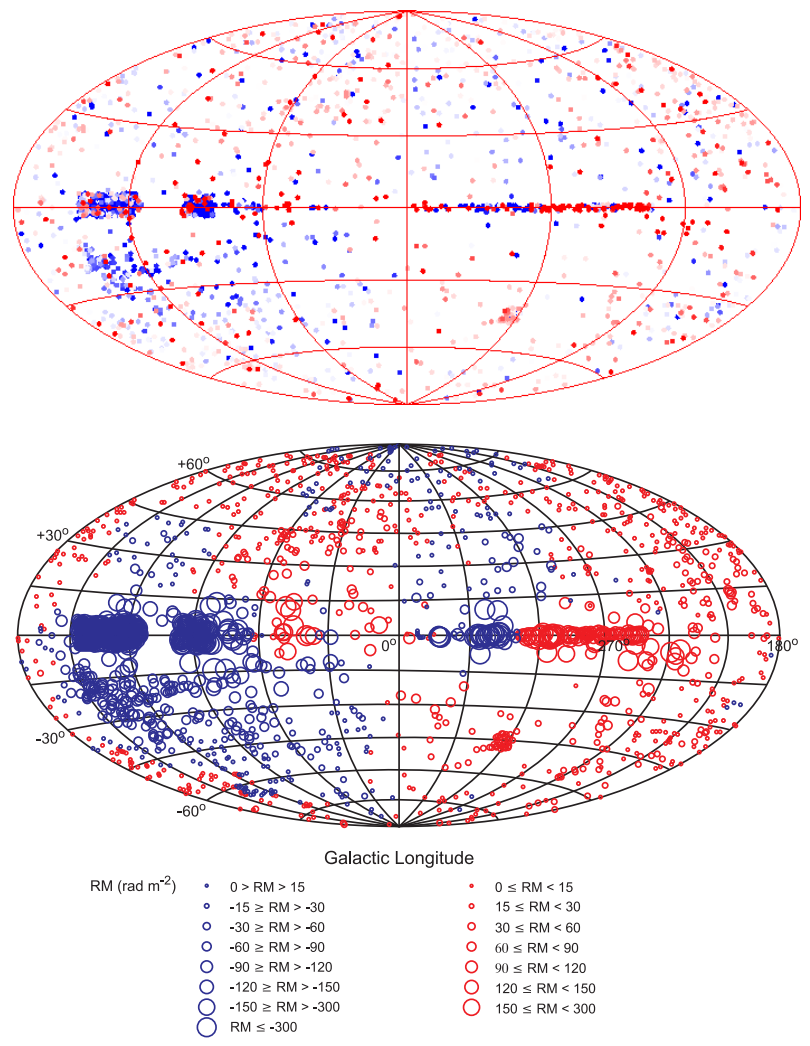

FIG. 2.- Upper panel: locations and RM signs of 2247 sources from KNM11. Red (blue) color corresponds to positive (negative) values of RM. Lower panel: the same data smoothed with a resolution of $21^{\circ}$ (adopted from Kronberg \& Newton-McGee (2011)).

sky map of the NVSS1 set is shown in Figure 1, which displays the location and RM sign of each source. The data have a large blind spot corresponding to declinations below $\sim-40^{\circ}$.

An advantage of the NVSS RM catalog is the large number of sources. However, observations being made in two close bands lead to relatively large individual RM errors. Also because in general RM is not always constant in $\lambda^{2}$, it can have modest variations over a wider range of $\lambda$. Thus an NVSS RM, measured in just one small wavelength range at $21 \mathrm{~cm}(\lambda=20.9-22.0 \mathrm{~cm})$, can differ from an RM measured over a wider wavelength

${ }^{1}$ We henceforth refer to NVSS rotation measure catalog as just NVSS. 
range which tends to average out fluctuations in $\operatorname{RM}\left(\lambda^{2}\right)$. Such systematic differences will add to the mean error of $\sim 11 \mathrm{rad} \mathrm{m}^{-2}$ quoted above by up to $\sim 13 \mathrm{rad} \mathrm{m}^{-2}$, and will contribute to the widths of the RM comparison plots with the results from the Kronberg \& Newton-McGee (2011) compilation (KNM11) shown in Figure 3 below. On the other hand, because $\lambda_{2}^{2}-\lambda_{1}^{2}$ is small, ambiguities of $n \pi$ in the NVSS RM's are unlikely to occur, especially at $|b|>10^{\circ}$.

It can be easily seen that the NVSS data set should be treated with care when considering the sources with large observed RMs, which mainly reside near the Galactic plane and in the region of inner Galaxy. In order to avoid this problem, we use the NVSS data only at Galactic latitudes $|b|>10^{\circ}$.

The second all-sky data set we used is the compilation by Kronberg \& Newton-McGee (2011) (2257 sources). It consists of $\sim 1500$ revised and statistically more accurate extragalactic radio source RMs of P. P. Kronberg et al. (in preparation). These are derived from polarization measurements made over a large "baseline" of $\lambda^{2}$. The remaining RM's are taken from the Canadian Galactic Plane Survey (Brown et al. 2003), the Southern Galactic Plane Survey (Brown et al. 2007), and two smaller published lists from Klein et al. (2003) (108 RMs) and (Mao et al. 2008) (68 RMs). The extragalactic radio sources in the KNM11 set were carefully observed at multiple frequencies and give RMs largely free from ambiguities. Errors of the RM measurements in the KNM11 set are typically of order $\sim 3 \mathrm{rad} \mathrm{m}^{-2}$. We use this set for control.

The sky map of the KNM11 RMs is shown in Figure 2, upper panel. The lower panel shows the same data smoothed over the circle of $21^{\circ}$ centered at the location of each source as described by Kronberg \& Newton-McGee (2011). One may observe a generally good agreement in the RM sky distributions of the NVSS and KNM11. This is particularly visible when comparing Figure 1 with the lower panel of Figure 2

The RM sky structure is rather peculiar. The regions of positive and negative RMs are roughly symmetric with respect to the Galactic plane in the outer Galaxy $90^{\circ}<l<270^{\circ}$ and anti-symmetric in the inner Galaxy. Note, however, that the boundary between positive and negative RMs in the inner Galaxy is displaced away from the Galactic plane (toward negative $b$ for $0<l<90^{\circ}$ ), that is, RMs do not change sign across the Galactic plane itself. This fact was observed, e.g., in Kronberg \& Newton-McGee (2011). This overall structure of RMs suggests, qualitatively, a Galactic field structure that consists of two components, with the disk field being symmetric in $b$ with respect to the Galactic plane and the halo field anti-symmetric.

Although the second data set is significantly smaller, it has much smaller individual RM errors and can be used in two ways. First, it allows for an independent evaluation of the quality of the NVSS data. In order to assess the accuracy of the NVSS RMs we identified, by positional cross-correlation, the sources that are common to both catalogs. We did it separately for the two regions $|b|>10^{\circ}$ and $|b|<10^{\circ}$. We found 1338 pairs and 306 pairs of sources, respectively, separated by less than $0^{\circ} .05$. For these sources, we built distributions of
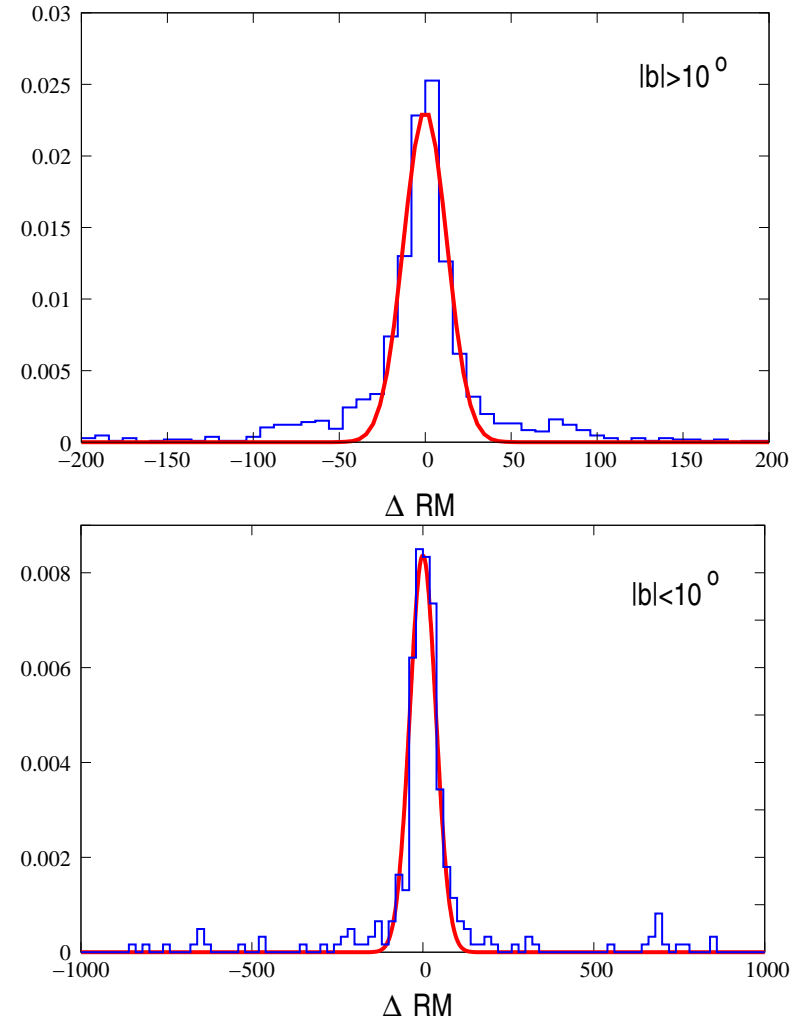

FIG. 3.- Distribution of differences of RMs as given by the NVSS catalog and by KNM11. The upper curve, for $|b|>10^{\circ}$, shows a Gaussian fit for the range $(-200,200) \mathrm{rad} \mathrm{m}^{-2}$. The lower curve, for $|b|<10^{\circ}$, shows the corresponding result for the full range $(-1000,1000) \mathrm{rad} \mathrm{m}^{-2}$.

differences between RMs given in the NVSS catalog and in the set by KNM11. The difference distributions are shown in Figure 3 ,

In both regions, the distributions of $\Delta \mathrm{RM}$ have peaks centered at zero and well fitted by Gaussians with dispersions $\sigma \simeq 13 \mathrm{rad} \mathrm{m}^{-2}$ and $\sigma \simeq 36 \mathrm{rad} \mathrm{m}^{-2}$, respectively. In the region $|b|>10^{\circ}$ the tails contain $\sim 25 \%$ of points and are confined within $\pm 100 \mathrm{rad} \mathrm{m}^{-2}$. The effective error introduced by these tails is of the order of the Gaussian part. Thus, in this region the NVSS data can be used for our purposes.

On the contrary, in the region $|b|<10^{\circ}$ the tails (containing similar fraction of points) are spread over a very wide range $\pm 900 \mathrm{rad} \mathrm{m}^{-2}$. Such tails would introduce errors in the low- $b$ bins which are too large to constrain the fit. For this reason we exclude the bins with $|b|<10^{\circ}$ from the NVSS fit, as will be described in Section 4 .

The KNM11 RM set provides statistically more accurate RMs in the Galactic plane, including areas where the NVSS data do not give sky coverage. This allows us to check whether the models which fit the NVSS data also fit the RM data in the Galactic plane.

\section{GMF AND ELECTRON DENSITY MODELS}

We adopt a general model of the GMF consisting of two different components: a disk field and a halo field (Prouza \& Smída 2003; Sun et al. 2008). Each of these two components is parameterized independently. According to the qualitative features of the RM distribution discussed above, for the most part of this work 


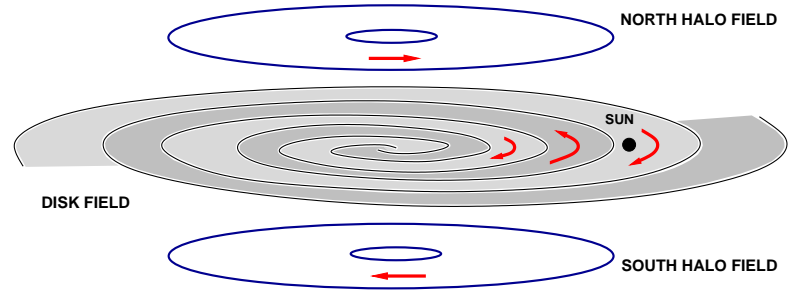

FIG. 4. - Sketch of the structure of the galactic magnetic field. The disk field in this sketch has reversals of the MF in adjacent arms. It is shown that the halo field is antisymmetric with respect to the galactic plane: in the solar neighborhood the direction of the halo field is anti-coincident with the direction of the disk field above the galactic plane and coincident below. We assumed that the disk field is symmetric with respect to the galactic plane, in the sense that the sign of the disk field is locally preserved across $b=0$.

we take the disk field to be symmetric with respect to the Galactic plane and the halo field anti-symmetric (other possibilities will be discussed in Section 5). The general layout of the model is shown in Figure 4

\subsection{The disk field}

We consider several disk GMF models. The first one is the widely used logarithmic spiral model, e.g., (Simard-Normandin \& Kronberg 1980; Han \& Qiao 1994; Stanev 1997; Tinyakov \& Tkachev 2002). There are two versions of this model depending on whether the direction of the field in two different arms is the same ${ }^{2}$ (axisymmetric, or ASS model) or opposite (bisymmetric, or BSS model). They are defined as follows:

$$
\begin{gathered}
B_{\theta}=B \cos p, B_{r}=B \sin p \\
B(r, \theta, z)=B(r)\left|\cos \left(\theta-b \ln \frac{r}{R_{\odot}}+\phi\right)\right| \exp \left(-|z| / z_{0}\right),
\end{gathered}
$$

or

$$
B(r, \theta, z)=B(r) \cos \left(\theta-b \ln \frac{r}{R_{\odot}}+\phi\right) \exp \left(-|z| / z_{0}\right),
$$

for ASS and BSS models, respectively. Here

$$
\begin{gathered}
\phi=b \ln \left(1+\frac{d}{R_{\odot}}\right)-\frac{\pi}{2}, \\
b \equiv 1 / \tan p,
\end{gathered}
$$

where $p$ is the pitch angle and $d$ is the distance to the first field reversal. Negative $d$ means that the nearest reversal occurs in the direction to the Galactic center, positive corresponds to the opposite direction. $R_{\odot}$ is the distance to the Galactic Center (we adopt $R_{\odot}=8.5 \mathrm{kpc}$ in this paper). The amplitude of the GMF is a function of the radial coordinate $r$,

$$
B(r)= \begin{cases}B_{0} \frac{R_{\odot}}{r \cos \phi}, & r>R_{c}, \\ B_{0} \frac{R_{\odot}}{R_{c} \cos \phi}, & r<R_{c},\end{cases}
$$

${ }^{2}$ In the work of Jansson et al. (2009) this model is dubbed as DSS-Disymmetric Spiral. where $R_{c}$ is the radius of the central region where the disk field is assumed to have constant magnitude. In the course of the simulations, the parameters were varied within the following ranges:

\begin{tabular}{l|l|l} 
parameter & $\min$ & $\max$ \\
\hline$R_{c}$ & $3 \mathrm{kpc}$ & $6 \mathrm{kpc}$ \\
$z_{0}$ & $0.5 \mathrm{kpc}$ & $1.5 \mathrm{kpc}$ \\
$d$ & $-1.4 \mathrm{kpc}$ & $1.4 \mathrm{kpc}$ \\
$p$ & $-15^{\circ}$ & $15^{\circ}$ \\
\hline
\end{tabular}

The second model of the disk field was the axisymmetric field with reversals in concentric rings. It was taken from Sun et al. (2008):

$$
\begin{gathered}
B(r, \theta, z)=D_{1}(r, z) D_{2}(r) \\
D_{1}(r, z)= \begin{cases}B_{0} \exp \left(-\frac{r-R_{\odot}}{R_{0}}-\frac{|z|}{z_{0}}\right), & r>R_{c} \\
B_{0} \exp \left(-\frac{|z|}{z_{0}}\right), & r \leq R_{c}\end{cases} \\
D_{2}(r)= \begin{cases}+1, & r>7.5 \mathrm{kpc}, \\
-1, & 6 \mathrm{kpc}<r \leq 7.5 \mathrm{kpc}, \\
+1, & 5 \mathrm{kpc}<r \leq 6 \mathrm{kpc}, \\
-1, & r \leq 5 \mathrm{kpc},\end{cases}
\end{gathered}
$$

where $R_{0}$ is radial scale of the MF. We studied the following parameter space:

\begin{tabular}{l|l|l} 
parameter & $\min$ & $\max$ \\
\hline$R_{c}$ & $4.5 \mathrm{kpc}$ & $5.5 \mathrm{kpc}$ \\
$R_{0}$ & $8 \mathrm{kpc}$ & $15 \mathrm{kpc}$ \\
$z_{0}$ & $0.5 \mathrm{kpc}$ & $1.5 \mathrm{kpc}$ \\
\hline
\end{tabular}

We also varied slightly the radial positions of the inner MF reversals (the boundaries of regions of different sign in Equation (77) ) in the ranges $4.8--5.2 \mathrm{kpc}, 5.8-$ $-6.2 \mathrm{kpc}$ and $7.3--8.0 \mathrm{kpc}$.

The magnitude of the disk MF at the vicinity of the solar System $B_{0}$ was taken to be $2 \mu \mathrm{G}$. Note that, in the total GMF, the disk and halo components will be combined with arbitrary coefficients (to be determined by fitting), so the choice $B_{0}=2 \mu \mathrm{G}$ at this point is merely a matter of definition.

\subsection{The halo model}

The basic halo model was taken from (Prouza \& Smída 2003; Sun et al. 2008):

$$
\begin{gathered}
B_{\theta}^{H}(r, z)= \\
B_{0}^{H}\left[1+\left(\frac{|z|-z_{0}^{H}}{z_{1}^{H}}\right)^{2}\right]^{-1} \frac{r}{R_{0}^{H}} \exp \left(1-\frac{r}{R_{0}^{H}}\right),
\end{gathered}
$$

where the direction of the field is reversed in the Southern hemisphere (Brandenburg et al. 1992; Han et al. 1997). We repeat here the following definitions from Sun et al. (2008): $B_{0}^{H}$ is the halo MF strength, $R_{0}^{H}$ is its radial scale, parameter $z_{0}^{H}$ defines the vertical position of the halo, $z_{1}^{H}$ is its vertical scale; this scale could differ in directions to and away from the galactic plane, $|z|<$ $z_{0}^{H}$ and $|z|>z_{0}^{H}$ respectively. We will refer to these parameters as $z_{1(1)}^{H}$ and $z_{1(2)}^{H}$, correspondingly. Here and below the index " $\mathrm{H}$ " denotes parameters belonging to the 
halo. The original values of the parameters in the model of Sun et al. (2008) were: $B_{0}^{H}=10 \mu \mathrm{G}, R_{0}^{H}=4 \mathrm{kpc}$, $z_{0}^{H}=1.5 \mathrm{kpc}$ and $z_{1}^{H}=0.2 \mathrm{kpc}$ for $|z|<z_{0}^{H}$, otherwise $z_{1}^{H}=0.4 \mathrm{kpc}\left(z_{1(1)}^{H}=0.2 \mathrm{kpc}\right.$ and $\left.z_{1(2)}^{H}=0.4 \mathrm{kpc}\right)$. We also tested halo models with slightly different radial profiles:

$$
\begin{gathered}
B_{\theta}^{H}(r, z)= \\
B_{0}^{H}\left[1+\left(\frac{|z|-z_{0}^{H}}{z_{1}^{H}}\right)^{2}\right]^{-1} \exp \left(-\left(\frac{r-R_{0}^{H}}{R_{0}^{H}}\right)^{2}\right),
\end{gathered}
$$

and

$$
\begin{gathered}
B_{\theta}^{H}(r, z)= \\
B_{0}^{H}\left[1+\left(\frac{|z|-z_{0}^{H}}{z_{1}^{H}}\right)^{2}\right]^{-1} \exp \left(-\left|\frac{r-R_{0}^{H}}{R_{T}}\right|\right) .
\end{gathered}
$$

We investigated the following parameter space:

\begin{tabular}{l|l|l} 
parameter & $\min$ & $\max$ \\
\hline$R_{0}^{H}$ & $3.5 \mathrm{kpc}$ & $15 \mathrm{kpc}$ \\
$R_{T}$ & $1 \mathrm{kpc}$ & $5 \mathrm{kpc}$ \\
$z_{0}^{H}$ & $0.8 \mathrm{kpc}$ & $3.5 \mathrm{kpc}$ \\
$z_{1}^{H}$ & $0.2 \mathrm{kpc}$ & $0.4 \mathrm{kpc}$ \\
$z_{1(2)}^{H}$ & $0.3 \mathrm{kpc}$ & $0.5 \mathrm{kpc}$ \\
\hline
\end{tabular}

Given that the halo field has not been firmly established as entirely azimuthal, we further studied the basic halo model, varying the pitch angle within the range $-10^{\circ}<$ $p^{H}<10^{\circ}$.

\subsection{The electron density}

Calculation of the RMs, Equation (2), requires a knowledge of the Galactic electron density $n_{e}$. In this paper we adopted the NE2001 model developed by Cordes \& Lazio (2002). However, there is mounting evidence that the original vertical scale of this model is insufficient and pulsar observations are better described by the electron density with larger vertical scale (Gaensler et al. 2008). We tried both the original $\left(z_{h}=\right.$ $0.95 \mathrm{kpc}, n_{e}=0.03 \mathrm{~cm}^{-3}$ at $\left.z=0\right)$ and modified versions $\left(z_{h}=1.8 \mathrm{kpc}, n_{e}=0.014 \mathrm{~cm}^{-3}\right.$ at $\left.z=0\right)$ of NE2001.

The preliminary simulations with both versions of NE2001 were made with a coarse grid of GMF parameters. The original model performed systematically worse than the modified one, so we proceeded to the full simulation with the modified version of NE2001. The results presented below were obtained with this modified version. Apart from the test of two vertical scales as described in Section 5, we did not vary any other parameters of NE2001 during the fits.

\section{METHOD}

\subsection{Binning}

RMs of individual sources are affected by uncertainties due to the intrinsic source contributions to the RM and by small-scale fluctuations of the GMF. The effect of the coherent component of the GMF can be revealed by averaging over a large number of nearby sources. The angular bin size should be small enough so that variations of the coherent component of GMF within the

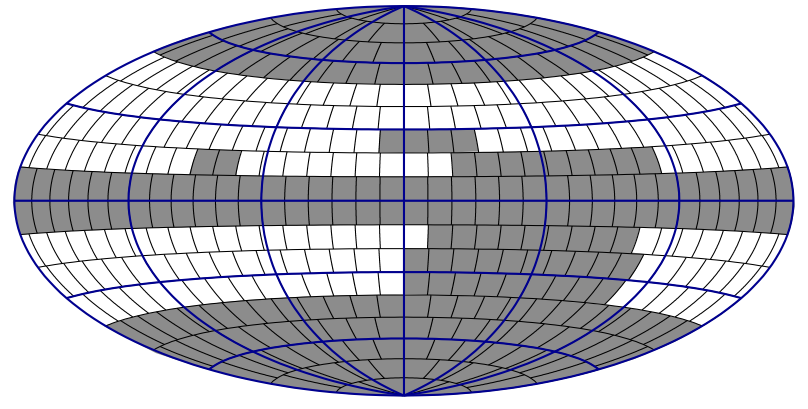

FIG. 5.- Binned celestial sphere. The bins marked in gray are excluded from the fit (see Appendix A for details).

bin can be neglected, and it should include a sufficient number of sources to suppress source-to-source fluctuations. Applying these requirements, we chose the following binning: the celestial sphere is divided into 18 bands of $10^{\circ}$ width, from $-90^{\circ}$ to $+90^{\circ}$ in Galactic latitude $b$. Each band is further divided into bins so that individual bins would have roughly equal area, about 100 $\mathrm{deg}^{2}$, as shown in Figure 5. So, there are 36 bins in the 9 th band $\left(0^{\circ}<b<10^{\circ}\right)$, but only four in the first one $\left(80^{\circ}<b<90^{\circ}\right)$. The total number of bins is equal to 422. After averaging, all the NVSS data are reduced to 422 values of average RM in each bin. No outliers were removed at this stage. Figures [6] and 7 shows the averaged RMs in the eight bands with $10^{\circ}<b<50^{\circ}$ and $-50^{\circ}<b<-10^{\circ}$, respectively. The coherent structure is clearly visible.

The GMF models were treated in a similar way. For each model we simulated 20,000 values ${ }^{3}$ of $\mathrm{RMs}$ at randomly chosen locations and then applied the same binning and averaging procedure to these sets. The resulting averaged RMs were compared to observations.

A somewhat different procedure was used for the set of sources from KNM11. To check the disk field, we used the sources residing within $1^{\circ}$ from the galactic plane. As their number is more limited (about 240) and they are located irregularly within their respective bins $\left(10 \mathrm{deg}^{2}\right.$ in this case), we simulated model RM values in their exact locations to avoid an additional error that would be produced by different distributions of simulated and real sources within the bin.

\subsection{Errors}

Important for this analysis is the weighting of different bins or, equivalently, the bin-by-bin estimation of errors. There are several sources of scatter of individual RMs within the bin: internal RM of the source, an anomalous extragalactic RM component, random component of the Galactic field, variation of the regular GMF over the bin, fluctuations of the Galactic electron density $n_{e}$, and the measurement error. All these factors contribute to deviations of the observed average value of $\mathrm{RM}$ from that predicted by the GMF model, and determine the error that has to be assigned to the bin.

If all the above factors were random, it would be possible to estimate the error by statistical means. Note, however, that some of these factors (e.g., random com-

3 We checked that increase in number of simulated sources from 20,000 to 40,000 does not lead to any significant changes. 

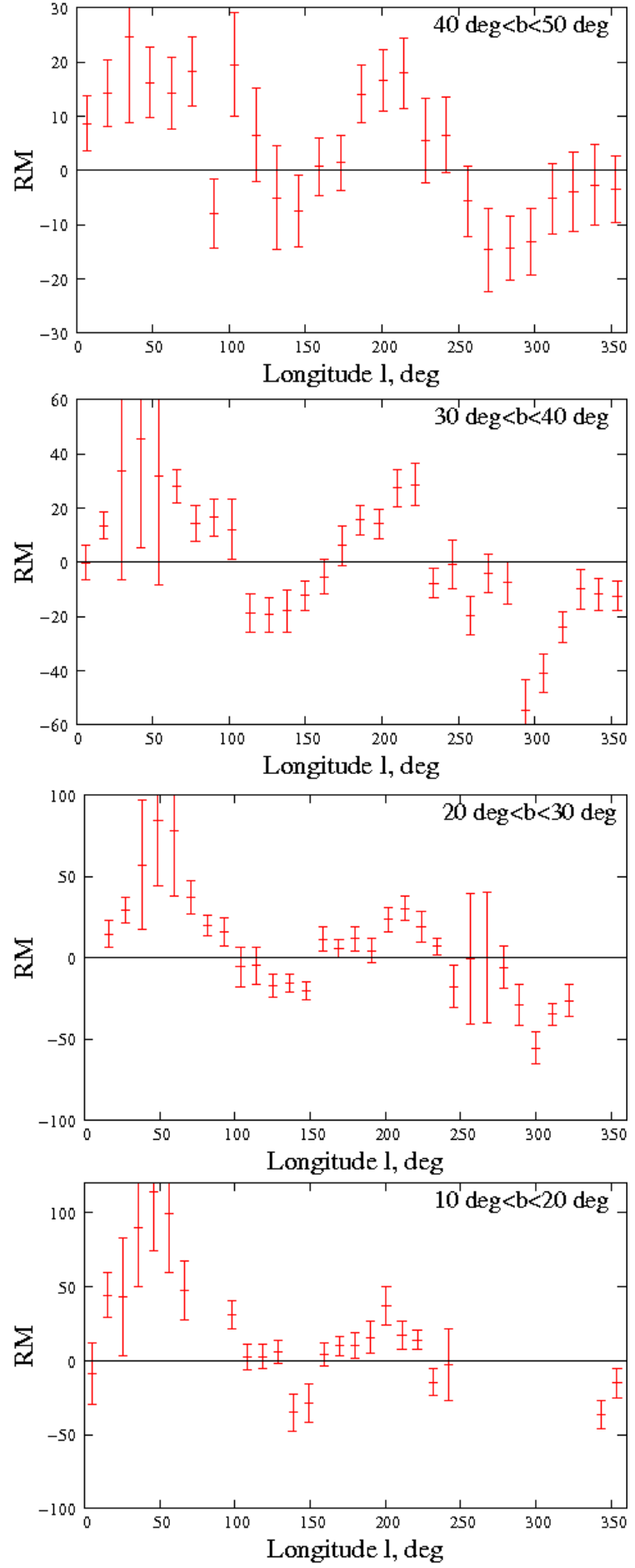

FIG. 6.- Averaged RMs in four bands $10^{\circ}<b<50^{\circ}$. The error bars show the errors used in the fit.

ponent of GMF with coherence length comparable to the bin size, fluctuations of $n_{e}$ ) may act coherently over the bin. These factors are much more difficult to treat rigorously. We therefore take a phenomenological approach and assume that these factors produce an error in the bin that is some fraction of the root mean square (rms) of the RMs in that bin. In Appendix $\mathrm{A}$ we develop a toy model where such proportionality takes place.

Taking account of this complication, we have estimated
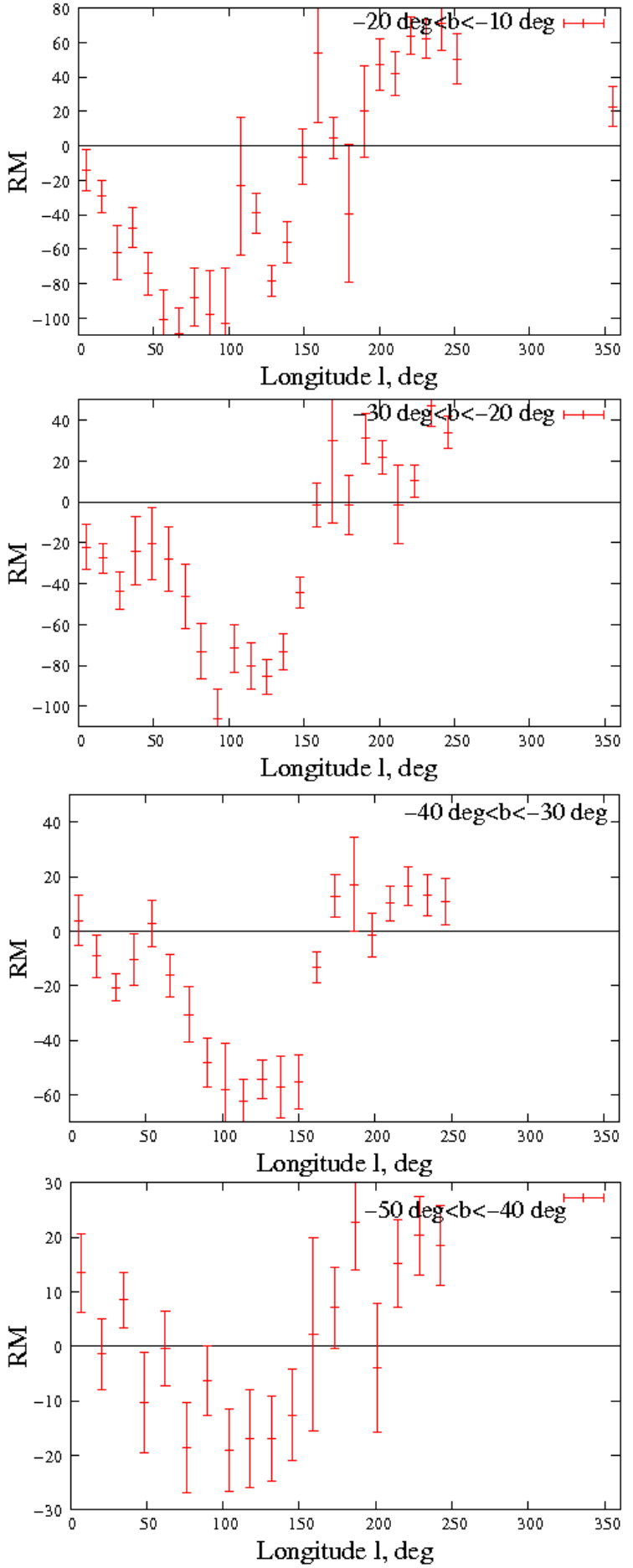

FIG. 7.- Averaged RMs in four bands $-50^{\circ}<b<-10^{\circ}$. The error bars show the errors used in the fit.

the errors of RMs in the individual bins as follows. By considering the sources in the directions of north and south Galactic poles where the effect of the regular MF is expected to be suppressed, the dispersion in the observed RMs of quasars is estimated as $\sigma_{\mathrm{qso}}=15 \mathrm{rad} \cdot \mathrm{m}^{-2}$ (see Appendix A). If the rms $\sigma_{i}$ of RMs in the $i$ th bin did not exceed this value, we set the error of average RMs to $\delta_{i}=\sigma_{\mathrm{qso}} / \sqrt{N_{i}}$, where $N_{i}$ is the number of sources in the bin. The reason is that in this case there is no indication 
of coherence effects, so one may assume that the error is due to stochastic factors such as the internal scatter, measurement error, and short-scale random MFs.

In the opposite case we assume that the variance in the observed RMs is (partly) due to the presence of some unknown features that could act coherently over the bin, but cannot be accurately modeled. We assigned the error of $\sigma_{i} / 3$ to these bins, where the factor $1 / 3$ is taken from the toy model of Appendix A. We have checked that the best-fit parameters do not change significantly when this factor is varied in the range $1 / 2-1 / 4$. This uncertainty in the absolute normalization of errors we cannot reduce further.

After a bin-by-bin examination, we have increased the errors in bins where there are clearly visible anomalies in the observed data (see the list of these bins below in the Appendix A . These problems are: (1) existence of small cluster of sources with RMs sharply different from the surrounding values (2) a strongly irregular distribution of the observed sources across the bin (due to, e.g., deep surveys within small regions) that could bias the average compared to the case of a random distribution of the simulated sources, and (3) the presence of sources with absolute values of RMs larger than $300 \mathrm{rad} \mathrm{m}^{-2}$ which should be treated with suspicion due to the $n \pi$ ambiguity in the definition of polarization angle. The total number of bins with errors increased by hand is about $10 \%$. The algorithm is described in Appendix A

Some bins were completely removed from the analysis. First, these are the bins in polar regions with $b>50^{\circ}$ (50 bins) and $b<-40^{\circ}$ (77 bins). In these regions the model values of RM are small because the projection of the MF onto the line of sight is suppressed (recall that in our model both disk and halo fields are parallel to the Galactic plane regardless of the parameters). On the other hand, there is strong evidence that some sort of 'screen' is present in the direction of high latitudes (Stil et al. 2011) which may provide a dominant contribution in these regions. The averaged $\mathrm{RMs}$ in polar regions, together with the assigned errors, are shown in Figures 13, and 14 where some bias toward positive values of RMs can indeed be seen.

Second, all bins with $|b| \leq 10^{\circ}$ were excluded because we could not trust the accuracy of the NVSS values in this region as explained in Section 2. Finally, we excluded all bins where the number of observed sources was smaller than 30 (this primarily concerns bins overlapping with the blind spot). Figure [5] shows the excluded bins in gray.

A similar prescription was adopted for the analysis of the $1^{\circ}$ strip along the Galactic plane using the RM set by KNM11, with the following changes. As the area in this case is 10 times smaller $\left(\sim 10 \mathrm{deg}^{2}\right.$ instead of $\left.100 \mathrm{deg}^{2}\right)$ we set our lower limit to three sources in the bin. Also, we increased the assigned error value to $\sigma_{i}$ instead of $\sigma_{i} / 3$, thus attempting to reproduce the enhanced uncertainty in the disk due to the presence of a large number of local structures and very long propagation path length inside the disk (of order of tens of $\mathrm{kpc}$ ). In the $1^{\circ}$ strip, after visual examination, we removed 20 sources out of total number of 259. RM values of the removed sources clearly demonstrate large differences with RMs of neighboring sources.

\subsection{Fitting procedure}

The best values of the GMF parameters were identified by the maximum likelihood method. This amounts to minimizing the value of the $\chi^{2}$ defined as follows,

$$
\chi^{2}=\sum_{i=1}^{\mathrm{Ntot}} \frac{\left(\mathrm{RM}_{\mathrm{obs}}-\mathrm{RM}_{\mathrm{sim}}\right)_{\mathrm{i}}^{2}}{\delta_{i}^{2}},
$$

where $N_{\text {tot }}$ is the total number of bins that we have included in the fit, $\left(\mathrm{RM}_{\mathrm{obs}}-\mathrm{RM}_{\text {sim }}\right)_{\mathrm{i}}$ is the difference between the observed and simulated average RM values for the $i$-th bin and $\delta_{i}$ is the adopted error of the observed average.

Since the RMs (Equation (2)) are linear in the MF, the total RM of a given source is a simple sum of the disk and halo contributions. Making use of this property, we separately simulated more than 30,000 halo RM maps and 10,000 disk RM maps, corresponding to different parameters varied within the ranges described in Section 3. At this stage the strengths of the disk and halo components were held fixed and set to values given in Section 3. Then each disk configuration was combined with each halo configuration with two arbitrary coefficients $\alpha$ and $\beta$. For a global GMF model thus obtained, the value of $\chi^{2}$ in Equation (11) is a quadratic polynomial in $\alpha$ and $\beta$, whose minimum with respect to $\alpha$ and $\beta$ we found analytically. The resulting values of $\alpha$ and $\beta$ determined the strength of the disk and halo fields.

The parameters of the GMF field were varied, within the ranges given in Section [3] by two different methods: by uniformly covering a given parameter range with a regular grid (grid-type simulation) and by randomly picking parameter values from a uniform distribution over the desired range (Monte Carlo type simulations). The disk field was simulated using the grid method, whereas for the halo we used both methods in roughly equal proportion.

Because of the uncertainty in the absolute normalization of $\chi^{2}$ resulting from uncertainties in the error estimation (see Section 4.2), we did not combine the Northern and Southern Galactic hemispheres in a single fit. The two hemispheres do not come on equal footing because of the blind spot in the Southern sky. Instead, our general fitting strategy was as follows. First we performed the fit using the NVSS data for the Northern hemisphere only, thus obtaining the preliminary set of models. Next, we fitted the NVSS data in the Southern hemisphere. We chose only the models that performed well in both tests and had the same value of the disk field. Note that this allowed for different halo fields in the Northern and Southern hemispheres. In these tests we imposed the additional constraint that $|\mathbf{B}|$ be greater than $1.8 \mu \mathrm{G}$ in both hemispheres, to conform with the results of other observations. Models were selected as acceptable if they satisfied the following requirement,

$$
\chi^{2} / \chi_{\min }^{2}=\chi_{\text {red }}^{2} / \chi_{\text {red,min }}^{2}<1+\sqrt{2 / \mathrm{N}_{\text {d.o.f. }}},
$$

where $\chi_{\min }^{2}\left(\chi_{\text {red,min }}^{2}\right)$ is a minimal value of $\chi^{2}\left(\chi_{\text {red }}^{2}\right)$. These are the models having parameters within $\sim 1 \sigma$ from the best-fit ones. Note that the criterion (12) is insensitive to the absolute normalization of the errors. We choose such a criterion to reduce the effect of the ambiguities in the error estimation discussed in Section 4.2 . 


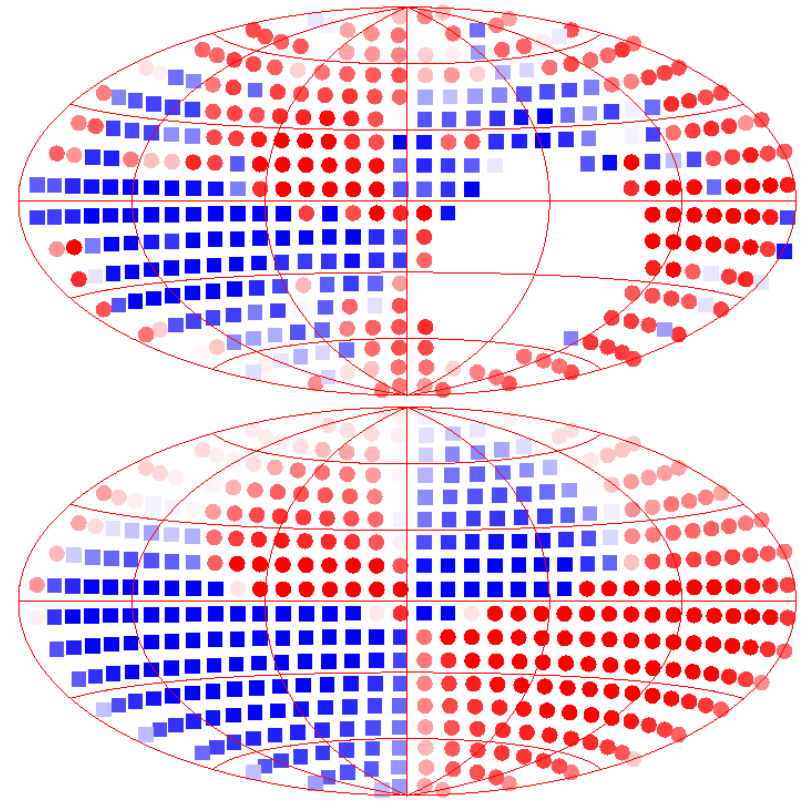

FIG. 8. - Average rotation measures in bins. Red circles (blue squares) represent positive (negative) RMs. The color intensity reflects the absolute magnitude. Top: NVSS data. Bottom: bestfit model.

Independently, we fitted the sources from the compilation by KNM11 in the narrow strip of the galactic plane $|b|<1^{\circ}$. This imposed additional constraints on the structure of the field in the thin disk to which our fit of NVSS data is insensitive. Requiring that fits to both the NVSS and KNM11 data be good, we performed the final selection of the models.

Overall, we required that a successful model passes three separate fits: to the NVSS data in the Northern and Southern hemispheres, and to the KNM11 data in the disk. Note that this is, in general, more restrictive than performing a single combined fit.

\section{RESULTS}

Figure 8 shows a comparison between one of the best fit models and the binned NVSS data. Positive average RMs are shown by red circles, and negative by blue squares. The intensity of the color indicates the absolute value of RM. It is seen that the general structure of the field is reproduced quite well.

The results of the fits are as follows:

North. The absolute minimum value of $\chi^{2}$ was equal to 232 for 104 degrees of freedom (dof), $\chi_{\text {red }}^{2}=2.23$. A bin-by-bin comparison between the data and the best-fit model is shown in Figure 9. Parameter ranges that were obtained from the fits are presented in Table 4. The fit was most sensitive to the pitch angle $p$. The vertical scale of the disk MF $z_{0}$ and the distance to the nearest reversal $d$ affect the fit less. There was no significant influence of the $R_{c}$ parameter, so we excluded it from further studies fixing its value at $R_{c}=5 \mathrm{kpc}$. The same concerns the parameters $z_{1(1)}^{H}$ and $z_{1(2)}^{H}$ which were fixed at $z_{1(1)}^{H}=0.25 \mathrm{kpc}$ and $z_{1(2)}^{H}=0.4 \mathrm{kpc}$.

We could not make a successful fit using the disk component only. The addition of the halo component decreases $\chi_{\min }^{2}$ by a factor of 1.5. Due to a weaker effect of the halo MF, its parameters are estimated with larger
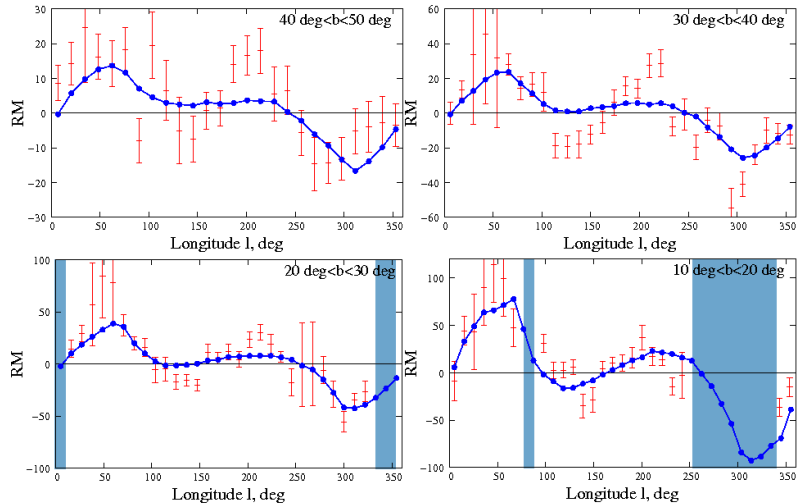

Fig. 9.- Best-fit model (blue solid line) vs. NVSS data (red points) in the Northern hemisphere. Bins not included in the fit are shaded with light blue.

uncertainties than the disk ones. In addition, there is a degeneracy between the height of the halo $z_{0}^{H}$, the amplitude of the halo field and, to some extent, the radial size $R_{0}^{H}$. Even unrealistically large values of the halo field of order tens of $\mu \mathrm{G}$ could give an acceptable fit if the halo is placed at a large distance from the galactic plane where the concentration of the electrons greatly decreases (cf. Figure 12). There is also a degeneracy between the vertical scales of the GMF model (both halo and disk) and the vertical scale of the electron density (recall that the latter was held fixed during the fits).

The strength of the disk field corresponding to the allowed set of models resides in the $1.8--2.8 \mu \mathrm{G}$ range, largely concentrating around $2.0 \mu \mathrm{G}$. The amplitude of the allowed halo field is in the range $2--12 \mu \mathrm{G}$. Note that the halo field strength is constrained by the synchrotron radio-emission from the population of Galactic relativistic electrons (Haslam et al. 1982). These constraints favor halo fields with the magnitude in the range $2--5 \mu \mathrm{G}$.

Ring models of the disk field performed rather poorly in the Northern hemisphere no matter what was the halo field, giving the best value of $\chi_{\min }^{2}=288$ for 101 dof, which corresponds to $\chi_{\text {red }}^{2}=2.85$ as compared to $\chi_{\text {red }}^{2}=2.23$ for the best-fit model with the spiral disk. Thus, these models are disfavored by the NVSS data, confirming the earlier finding of Simard-Normandin \& Kronberg(1980).

Finally, our attempts to improve the quality of the fit by introducing a non-zero halo pitch angle were unsuccessful: there was no significant change of $\chi^{2}$ when nonzero pitch angle of the halo field was varied within the range $\left[-10^{\circ}, 10^{\circ}\right]$. We also could not improve the fit by choosing the alternative model of the halo described by Equations (9) and (10).

South. The data from the Southern hemisphere are more sparse because of the blind spot, and show more scatter, so the fits are generally worse. The best-fit ASStype model gives $\chi^{2}=243$ for 65 dof, which corresponds to $\chi_{\text {red }}^{2}=3.74$. The results for the best-fit BSS model are $\chi^{2}=235$ for 65 dof $\left(\chi_{\text {red }}^{2}=3.62\right)$. The bin-by-bin comparison between the data and the best-fit model in the Southern hemisphere is shown in Figure 10.

Disk. Finally, we fitted the RMs from KNM11 at very low latitudes $|b|<1^{\circ}$. The best-fit ASS-type model gives 

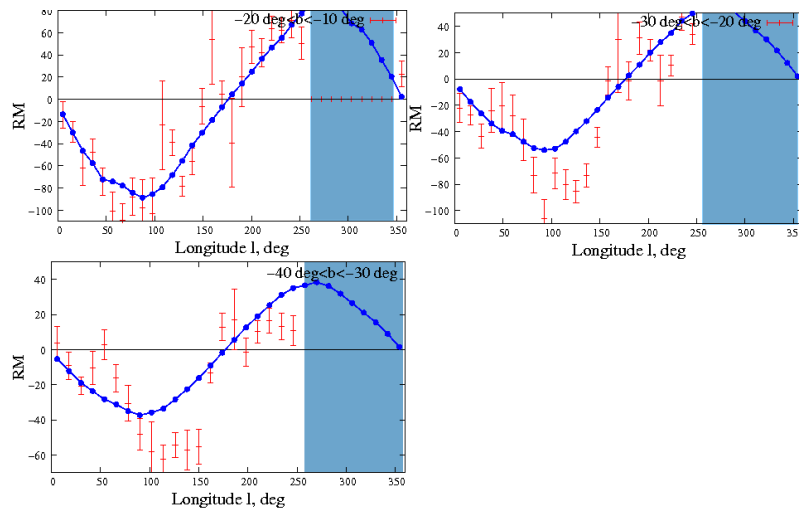

FIG. 10.- Best-fit model (blue solid line) vs. NVSS data (red points) in the Southern hemisphere. Bins not included in the fit are shaded with light blue.
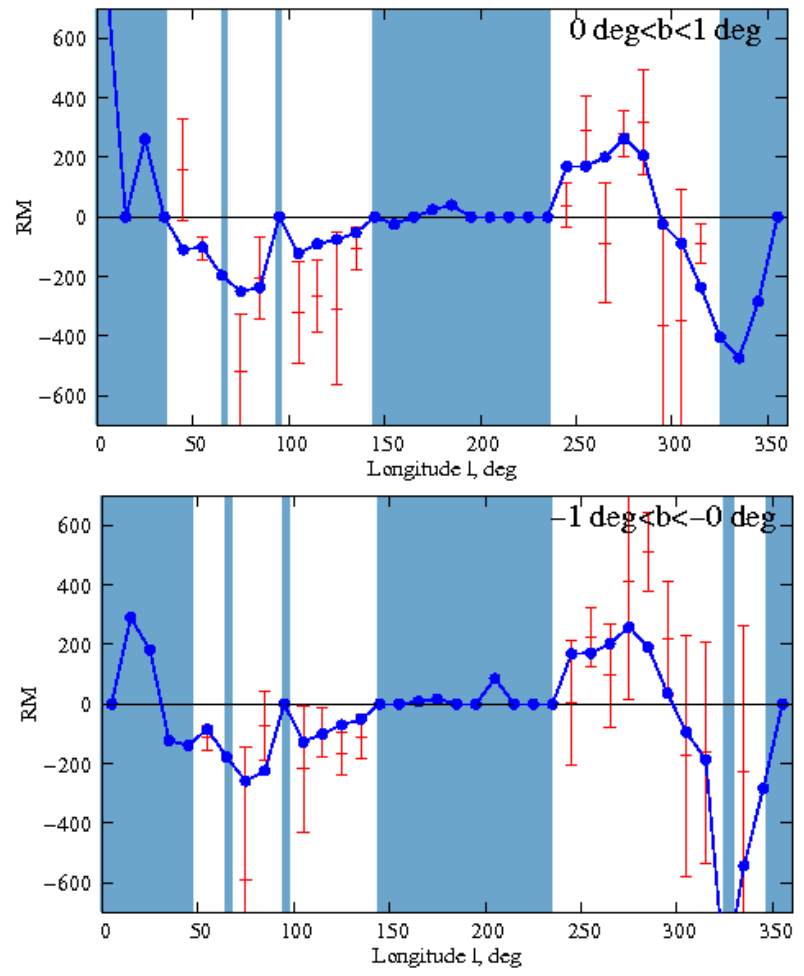

Fig. 11.- Comparison between the best-fit model and the data from KNM11 in the strip $-1^{\circ}<b<1^{\circ}$. Areas not included into the fit are shaded with light-blue.

$\chi^{2}=30$ for 29 dof $\left(\chi_{\text {red }}^{2}=1.03\right)$, while the best-fit BSS model gives $\chi^{2}=37$ for 29 dof $\left(\chi_{\text {red }}^{2}=1.28\right)$. The comparison between these data and the best-fit model is shown in Figure 11. The results are fully compatible with the other fits. They also agree with the results of Kronberg \& Newton-McGee (2011). Unfortunately, there is not enough information about the region around the direction to the Galactic center where the discrepancy between predictions of different models is the largest. This reduces the discriminative power of the fit. Nevertheless, the fit was very sensitive to the field strength in the disk, so its results impose most stringent constraints on this parameter. Also, it was shown by Kronberg \& Newton-McGee (2011) that the KNM11 data favor the pitch angle near the disk of $-5 .^{\circ} 5$. Taking this constraint into account allows us to exclude positive pitch angles, which is not possible from the fits to the NVSS data alone.

In both north and south fits of the NVSS data, the reduced $\chi^{2}$ values significantly exceed unity (especially in the Southern hemisphere). This could result either from our underestimation of errors, or from features in the data that cannot be accounted for by our models. Visual examination of the fits (Figures 9 and 10) shows that there exists a systematic discrepancy between the data and the best-fit models in the region $l \approx 130^{\circ}--220^{\circ}$ which corresponds to directions away from the Galactic center. The discrepancy is present in both Northern and Southern hemispheres.

In fact, the observed variation of $\mathrm{RM}$ in the outer Galaxy is too strong to be accounted for in any model that has a thick disk, or halo with a nearly tangential field in the outer Galaxy (i.e., small pitch angle) if the field magnitude decays away from the Galactic center. So, either these models are completely wrong in this region, or the large RMs are caused by some nearby anomaly in the MF or the electron density, which is sufficiently close to subtend a large angle of about $\sim 90^{\circ}$. The latter option is supported by the fact that the variation of RM which is responsible for this feature does not visibly decrease away from the Galactic plane, as it would if it were due to an additional galactic arm or another global (and more distant) feature. Interestingly, the anomaly in the nearby region (the so-called region A) was pointed out previously (Simard-Normandin \& Kronberg 1980).

For an additional check of this assumption we made a separate fit of RMs of the sources located in the direction of the inner Galaxy, i.e., at $-90^{\circ}<l<90^{\circ}$. Excluding this apparent anomaly should improve the fit and show the extent to which the best-fit parameters were affected by its presence. The results of this additional test are as follows.

North-inner. The best fit gives $\chi_{\min }^{2}=43$ for 46 dof, considerably better than for the full sky. The allowed values of parameters obtained from the restricted fit largely coincide with those obtained from the full fit. This indicates that the full fit is not dominated by the anomalous region.

South-inner. $\chi_{\min }^{2}=35$ for 22 dof. The quality of the fit has considerably increased as compared to the fit in the full range of longitudes, but is still worse than in the Northern hemisphere.

All our attempts to fit the outer Galaxy separately $\left(90^{\circ}<l<270^{\circ}\right)$, even those including an extra disk component, were unsuccessful in the sense that we could not get $\chi_{\text {red }}^{2}$ smaller than five. This confirms our conclusion that it is not possible to fit the anomalous region with the GMF models of the type considered here.

The results of individual fits are presented in Table 4 in the form of acceptable ranges of model parameters. It should be stressed that there are dependencies and degeneracies among them; Table 4 only shows the maximum variation of each parameter within the whole acceptable set of models. In other words, not every combination of the acceptable parameters gives an acceptable model. Parameters of the models which fit the data best and are in agreement with all fits are summarized in Ta- 
TABLE 1

\begin{tabular}{ccccc}
\hline \hline & $p$ & $z_{0}$ & $d$ & $B_{0}^{D}$ \\
$\mathrm{deg}$ & $\mathrm{kpc}$ & $\mathrm{kpc}$ & $\mu \mathrm{G}$ \\
\hline ASS & {$[-5,-4] \cup[3,7]$} & {$[0.7,1.2]$} & {$[-0.7,-0.4]$} & {$[1.8,2.2]$} \\
BSS & {$[-7,-3] \cup[5,8]$} & {$[0.8,1.2]$} & {$[-0.9,-0.5]$} & {$[1.8,2.4]$} \\
\hline
\end{tabular}

Note. - Ranges of parameters of the disk field corresponding to $\sim 1 \sigma$ deviation from the best fit to the NVSS data and compatible with the KNM11 data in the Galactic disk. Note that positive ranges of the pitch angle, although compatible with our fits, are strongly disfavored by the "shift-and-reflection" arguments of KNM11.

TABLE 2

\begin{tabular}{lccc}
\hline \hline & $\begin{array}{c}z_{0}^{H} \\
\mathrm{kpc}\end{array}$ & $\begin{array}{l}R_{0}^{H} \\
\mathrm{kpc}\end{array}$ & $\begin{array}{l}B_{0}^{H} \\
\mu \mathrm{G}\end{array}$ \\
& & & \\
& & North \\
vs. ASS & {$[1.2,2.4]$} & {$[6.0,15]$} & {$[4.0,12]$} \\
vs. BSS & {$[1.0,2.0]$} & {$[8.0,15]$} & {$[3.0,12]$} \\
& \multicolumn{3}{c}{ South } \\
vs. ASS & {$[1.0,2.5]$} & {$[3.5,15]$} & {$[1.5,6.0]$} \\
vs. BSS & {$[1.0,2.2]$} & {$[4.0,15]$} & {$[2.5,5.0]$}
\end{tabular}

Note. — The same as Table 1 but for the halo parameters.

\section{bles 11, and 2}

Finally, let us comment briefly on the other tests that we have made. First, as has been already mentioned in Section 3.3 , we have run preliminary simulations with the original, lower vertical scale of electron density. For the realistic strength of the disk field $B_{0}>1.8 \mu G$ and the original electron density, we found only $\chi^{2}$ values larger than 390 as compared to 232 for the best-fit case. If we vary the magnitude of the disk field, the value of $\chi^{2}$ becomes close to the best-fit value for field strengths $\sim 0.7 \mu G$, which is clearly unacceptable. In conclusion, our simulations disfavor the original vertical scale of the electron density.

Second, we have tested different combinations of symmetries (with respect to the Galactic plane) of the halo and disk fields in order to check whether the NVSS data alone favor the symmetric disk and antisymmetric halo as we have chosen in our model. With our fitting procedure which treats the Northern and Southern hemispheres independently, no additional simulations are required for this test. For instance, for an anti-symmetric disk field we simply have to require that the coefficients with which the disk contribution enters the Northern and Southern hemispheres are opposite in sign. We could not find a satisfactory fit neither with ASS nor with BSS anti-symmetric disk field, obtaining the values of $\chi^{2}$ more than four times larger than the best-fit value. In the case of the symmetric disk and symmetric halo, the best fit gives $\chi^{2} 2.5$ times larger than the best-fit model. Thus, the combination of the symmetric (with respect to $b$ ) disk and antisymmetric (with respect to $b$ ) halo is favored by the NVSS data.

\section{CONCLUSIONS}

The latest Faraday rotation data show an unmistakable coherent component of the RMs over the sky. The RMs are correlated in sign and value over the largest angular scales. Moreover, the distribution of RMs is correlated with the Galactic plane and the direction to the Galactic center. This strongly indicates the Galactic origin of this coherence and provides firm evidence for the existence of a regular GMF.

Apart from the Galactic plane region $|b| \lesssim 10^{\circ}$, the global pattern of the RMs has different symmetry with respect to the Galactic plane in the inner and outer Galaxy: symmetric outside and anti-symmetric inside (see Figure 8). This change of the symmetry cannot be achieved with a single (symmetric or antisymmetric) component of the GMF. At least two components- disk and halo - are required (Figure 4). Since the study of the MF in the disk shows that it does not change sign across the Galactic plane itself, the combination of the symmetric disk field and the anti-symmetric halo field is favored. We have demonstrated that such a combination, with properly chosen amplitudes of components, reproduces the observed pattern of RMs very well.

Several further conclusions can be made.

1. The cross check between the NVSS and the smaller KNM11 set shows that the quality of the NVSS is sufficient for our purposes all over the sky except, in the $|b|<10^{\circ}$ band around the Galactic plane.

2. There is a feature in the observed RMs near the direction to the Galactic anti-center that cannot be accounted for by models of the type considered in this paper (nearly tangential field decaying away from the Galactic center). This feature may be due to some unspecified local structure.

3. A ring model of the GMF is disfavored as compared to the model with logarithmic spiral arms. In the former case the best value of $\chi_{\text {red }}^{2}$ is a factor of $\geq 1.3$ larger than in the latter.

4. Our fits favor the increased vertical scale of the electron density distribution as proposed by Gaensler et al. (2008). We were unable to find any fit with the original vertical scale of NE2001 model and strong disk field. This is in agreement with the results of Sun \& Reich (2010).

5. Our fits favor somewhat smaller values of the pitch angle $p$ than have been discussed in the literature previously. These smaller values agree with the recent results of Kronberg \& Newton-McGee (2011) where the reference value of $p=-5 .{ }^{\circ} 5$ was obtained by a totally different method.

Our fitting procedure could not discriminate between the ASS and BSS models because a major contribution to the extragalactic source RMs (especially at $|b|>15^{\circ}$ ) comes from the near vicinity of the solar system; in this region both models behave quite similarly. A more detailed analysis of the Galactic plane region may be more sensitive to the differences between the ASS and BSS models.

Two "benchmark" models (one with the ASS and one with the BSS disk field) are presented in Table 3. These 
TABLE 3

BENCHMARK MODEL PARAMETERS.

\begin{tabular}{lcc}
\hline \hline & ASS & BSS \\
& & \\
\hline Disk: & & \\
$p$ & $-5^{\circ}$ & $-6^{\circ}$ \\
$z_{0}$ & $1.0 \mathrm{kpc}$ & $1.0 \mathrm{kpc}$ \\
$d$ & $-0.6 \mathrm{kpc}$ & $-0.6 \mathrm{kpc}$ \\
$B_{0}$ & $2.0 \mu \mathrm{G}$ & $2.0 \mu \mathrm{G}$ \\
$R_{c}$ & $5.0 \mathrm{kpc}$ & $5.0 \mathrm{kpc}$ \\
& & \\
Halo (North): & & \\
$z_{0}^{H}$ & $1.3 \mathrm{kpc}$ & $1.3 \mathrm{kpc}$ \\
$R_{0}^{H}$ & $8 \mathrm{kpc}$ & $8 \mathrm{kpc}$ \\
$B_{0}^{H}$ & $4 \mu \mathrm{G}$ & $4 \mu \mathrm{G}$ \\
$z_{1(1)}^{H}$ & $0.25 \mathrm{kpc}$ & $0.25 \mathrm{kpc}$ \\
$z_{1(2)}^{H}$ & $0.4 \mathrm{kpc}$ & $0.4 \mathrm{kpc}$ \\
& & \\
Halo (South): & & \\
$z_{0}^{H}$ & $1.3 \mathrm{kpc}$ & $1.3 \mathrm{kpc}$ \\
$R_{0}^{H}$ & $8 \mathrm{kpc}$ & $8 \mathrm{kpc}$ \\
$B_{0}^{H}$ & $2 \mu \mathrm{G}$ & $4 \mu \mathrm{G}$ \\
$z_{1(1)}^{H}$ & $0.25 \mathrm{kpc}$ & $0.25 \mathrm{kpc}$ \\
$z_{1(2)}^{H}$ & $0.4 \mathrm{kpc}$ & $0.4 \mathrm{kpc}$ \\
& & \\
\hline
\end{tabular}

models fit the RM data well and have values of parameters favored by other studies (not too small pitch angle $p$, not too strong halo field and the magnitude in the vicinity of the Earth close to $2 \mu \mathrm{G}$ ). They can be used, e.g., for propagation of cosmic rays through the Galaxy. At ultra-high energies, an accurate three-dimensional description of the Galactic magnetic field may be crucial for identification of extragalactic cosmic ray sources.

In this paper we used only the Faraday RMs of extragalactic radio sources. More precise understanding of the GMF can be achieved by taking into account of other data, also sensitive to the MF, such as RMs of pulsars, measurements of the galactic synchrotron radiation, etc. This would permit better constraints on the unknown quantities such as the three-dimensional electron density profiles and other properties of the ISM. It will also help to resolve some degeneracies inherent in our models.

\section{ACKNOWLEDGMENTS}

The authors are grateful to D.D. Sokoloff and JinLin Han for fruitful discussions. Also, we thank an anonymous referee for careful reading of the manuscript and valuable remarks. This work is supported by IISN project No. 4.4509.10 and the Natural Sciences and Engineering Research Council of Canada (NSERC), the Australian Research Council, the U.S. Department of Energy, and the use of NASA's Astrophysics Data System.

\section{APPENDIX}

\section{ERROR ASSIGNMENT. EXCLUDED BINS AND BINS WITH MANUALLY ASSIGNED ERRORS}

In our fitting procedure, each bin included in the analysis was characterized by an average RM and its error $\delta$. Here we describe the algorithm by which these errors were assigned on a bin-by-bin basis.

First, we estimated the intrinsic dispersion of RMs of extragalactic sources. To this end we investigated the regions on the sky where the influence of the GMF is expected to be suppressed. Since RMs are proportional to the MF component that is parallel to the line of sight, these are the regions around the Galactic poles. Considering the sources with $|b|>80^{\circ}$ we found that the dispersion of RMs of individual sources in these regions was about $\sigma_{\mathrm{qso}}=15 \mathrm{rad} \mathrm{m}^{-2}$. Comparing to Figure 3 we see that the dominant contribution to this number comes from the measurement errors. In the error assignment algorithm, we adopted the value of $\sigma_{\mathrm{qso}}$ as an indicator of whether a given bin is perturbed by effects other than the random scatter of sources.

If a bin showed the dispersion of RMs $\sigma_{i}$ smaller than $\sigma_{\text {qso }}$ we considered it unperturbed and assigned its error on a statistical basis. Since the average of a sample of $N$ elements varies around the true average with the width $\sigma / \sqrt{N}$, where $\sigma^{2}$ is the variance of the distribution, we assigned to such bins an error of $\delta_{i}=\sigma_{\mathrm{qso}} / \sqrt{N_{i}}, N_{i}$ being the number of sources in the bin. In practice, this situation occurs only for high-latitude bins.

In the opposite case, i.e., when the dispersion $\sigma_{i}$ was larger than $\sigma_{\mathrm{qso}}$, we considered the bin as perturbed by coherence effects. These may be due to, e.g., random MF with the coherence length comparable to the bin size, fluctuations of electron density, strong variation of the regular component across the bin, etc. In this case the statistical approach is not correct, and a precise determination of the error is difficult. We have assumed that the error in such bins is some fraction of the bin variance $\sigma_{i}$, which we have adopted to be $1 / 3$, so that the error in perturbed bins was taken $\delta_{i}=\sigma_{i} / 3$. We cannot rigorously derive this coefficient (although it can be motivated by a toy model presented below). Since this uncertainty propagates directly into the value of $\chi^{2}$, it results in some uncertainty of the absolute normalization of the latter. Note, however, that the ratios of $\chi^{2}$ are less affected by this uncertainty.

The following toy model shows how the proportionality between the error and the dispersion in the bin may come about. Let the random part of the MF consist of cells of a typical size $d \sim 100 \mathrm{pc}$, such that their contributions to RM have zero mean and the dispersion $\sigma_{0}$. If the cells were statistically independent, individual sources would have an RM dispersion given by $\sigma=\sigma_{0} \sqrt{N_{\text {tot }}}$, where $N_{\text {tot }}$ is the total number of cells along the line of sight (we have neglected the internal scatter). However, for sources in one bin a number $N_{\text {coh }}$ of nearby cells contributes coherently. If $N_{\text {coh }} \ll N_{\text {tot }}$ this will not change the dispersion $\sigma$ over the bin. The error, on the contrary, will be dominated by the coherent contribution, $\delta=\sigma_{0} \sqrt{N_{\text {coh }}}=\sigma \sqrt{N_{\text {coh }} / N_{\text {tot }}}$. Thus, the error and the dispersion are proportional to one another with the coefficient $\sqrt{N_{\text {coh }} / N_{\text {tot }}}$. The latter coefficient is purely geometrical. Taking $N_{\text {coh }} \sim 1 / \theta$, where $\theta$ is the bin size, and $N_{\text {tot }} \sim L / d$, where $L$ is the length of the trajectory inside GMF, we find $\delta \sim \sigma / 3$. Interestingly, similar conclusions (proportionality and a similar coefficient) are obtained by considering a perturbation caused by a close group of a few sources having similar RMs substantially different from the rest of the sources in the bin. 
As a last step, all bins were subject to visual scrutiny, with the result that some of the bins were completely removed from the analysis. These are the bins showing obvious pathologies like small number of sources or non-uniform distribution across the bin (e.g., some bins at the boundary of the NVSS blind spot), compact aggregates of sources with RMs drastically different from the surrounding value, etc. Finally, some bins with such pathologies were assigned large errors (2-3 times larger than prescribed by the above algorithm) by hand. This was done in cases where the corresponding bin might serve as a good discriminator between models, even with a large error. A constant error of $40 \mathrm{rad} \mathrm{m}^{-2}$ was used in this case. This value was chosen in such a way that these bins do not contribute significantly into the value of $\chi^{2}$, at least near the best-fit point. We have checked that the results of the fits do not depend on the precise choice of these errors. The number of such bins is about 10\%; they are easily identifiable in Figures 6 and 7 .

Here is the complete list of excluded bins and bins with manually assigned errors.

Northern hemisphere. An error of $40 \mathrm{rad} \mathrm{m}^{-2}$ was assigned to the bins in the region $30^{\circ}<l<60^{\circ}, 10^{\circ}<b<40^{\circ}$ (local structures described in (Wolleben et al. 2010)); to the bins in the area $-115^{\circ}<l<-90^{\circ}, 20^{\circ}<b<30^{\circ}$ (a prominent structure with large positive RMs, possibly related with the Gum nebula); to the bin located at $\left(15^{\circ}, 25^{\circ}\right)$ (strong non-uniformity: only 12 sources are located at $10^{\circ}<b<15^{\circ}$, while 38 at $15^{\circ}<b<20^{\circ}$ ). Excluded from the analysis were the bins in the area $-10^{\circ}<l<30^{\circ}, 20^{\circ}<b<30^{\circ}$ (unresolved structure in RM and a large positive spot at $\left(-10^{\circ}, 25^{\circ}\right)$ ); bins in the area $70^{\circ}<l<90^{\circ}, 10^{\circ}<b<20^{\circ}$ (large negative spot centered at $\left(80^{\circ}, 15^{\circ}\right.$ ); bin affected by Gum nebula at $\left(l=-100^{\circ}, b<20^{\circ}\right)$; the bin centered at $\left(-30^{\circ}, 15^{\circ}\right)$ (strongly non-uniform distribution). Southern hemisphere. An error of $40 \mathrm{rad} \mathrm{m}^{-2}$ was assigned to the bin $\left(105^{\circ},-15^{\circ}\right)$ (local structure resulting in positive excess ); to the bin $\left(165^{\circ},-15^{\circ}\right)$ (positive spot); to the bin centered at $\left(180^{\circ},-15^{\circ}\right)$ (negative spot near the anti-center direction); and to the bin $\left(170^{\circ},-25^{\circ}\right)$ (large positive spot). 
TABLE 4

RANGES OF DisK AND HALO PARAMETERS OF THE ACCEPTABLE MODELS

\begin{tabular}{|c|c|c|c|c|c|c|c|c|c|}
\hline & $\chi^{2}$ & $\chi_{\text {red }}^{2}$ & $\begin{array}{c}p \\
\operatorname{deg}\end{array}$ & $\begin{array}{c}z_{0} \\
\mathrm{kpc}\end{array}$ & $\begin{array}{c}d \\
(\mathrm{kpc})\end{array}$ & $\alpha$ & $\begin{array}{l}z_{0}^{H} \\
\mathrm{kpc}\end{array}$ & $\begin{array}{l}R_{0}^{H} \\
\mathrm{kpc}\end{array}$ & $\bar{\beta}$ \\
\hline \multicolumn{10}{|c|}{ North } \\
\hline $\begin{array}{l}\mathrm{A} \\
\mathrm{B}\end{array}$ & $\begin{array}{l}240 \\
232\end{array}$ & $\begin{array}{l}2.30 \\
2.23\end{array}$ & $\begin{array}{l}{[-10,-3]} \\
{[-6,-10]}\end{array}$ & $\begin{array}{l}{[0.5,1.3]} \\
{[0.5,1.3]}\end{array}$ & $\begin{array}{l}{[-1.1,-0.3]} \\
{[-1.1,-0.5]}\end{array}$ & $\begin{array}{l}{[0.9,2.0]} \\
{[0.9,1.5]}\end{array}$ & $\begin{array}{l}{[1.0,1.4]} \\
{[1.0,1.4]}\end{array}$ & $\begin{array}{l}{[4,15]} \\
{[6,15]}\end{array}$ & $\begin{array}{l}{[0.4,0.7]} \\
{[0.3,1.0]}\end{array}$ \\
\hline \multicolumn{10}{|c|}{ South } \\
\hline $\begin{array}{l}\mathrm{A} \\
\mathrm{B}\end{array}$ & $\begin{array}{l}243 \\
235\end{array}$ & $\begin{array}{l}3.74 \\
3.62\end{array}$ & $\begin{array}{l}{[-10,10]} \\
{[-10,10]}\end{array}$ & $\begin{array}{l}{[0.5,1.5]} \\
{[0.5,1.1]}\end{array}$ & $\begin{array}{l}{[-1.1,1.1]} \\
{[-1.0,1.0]}\end{array}$ & $\begin{array}{l}{[0.9,2.0]} \\
{[0.2,2.0]}\end{array}$ & $\begin{array}{l}{[1.0,3.5]} \\
{[1.0,3.5]}\end{array}$ & $\begin{array}{l}{[3.5,15]} \\
{[3.5,15]}\end{array}$ & $\begin{array}{l}{[0.2,2.0]} \\
{[0.2,2.0]}\end{array}$ \\
\hline \multicolumn{10}{|c|}{ Plane } \\
\hline $\begin{array}{l}\mathrm{A} \\
\mathrm{B}\end{array}$ & $\begin{array}{l}30 \\
37\end{array}$ & $\begin{array}{l}1.03 \\
1.27\end{array}$ & $\begin{array}{c}{[-7,10]} \\
{[-8,-3] \cup[5,8]}\end{array}$ & $\begin{array}{l}{[0.5,1.4]} \\
{[0.5,1.5]}\end{array}$ & $\begin{array}{l}{[-1.4,1.4]} \\
{[-0.8,1.2]}\end{array}$ & $\begin{array}{l}{[0.9,1.1]} \\
{[1.0,1.2]}\end{array}$ & & & \\
\hline \multicolumn{10}{|c|}{ North-inner } \\
\hline $\begin{array}{l}\mathrm{A} \\
\mathrm{B}\end{array}$ & $\begin{array}{l}43 \\
45\end{array}$ & $\begin{array}{l}0.93 \\
0.98\end{array}$ & $\begin{array}{l}{[-5,6]} \\
{[-7,9]}\end{array}$ & $\begin{array}{l}{[0.5,1.2]} \\
{[0.5,1.4]}\end{array}$ & $\begin{array}{l}{[-1.0,0.9]} \\
{[-0.6,1.1]}\end{array}$ & $\begin{array}{l}{[0.9,3.0]} \\
{[0.9,1.1]}\end{array}$ & $\begin{array}{l}{[1.2,2.4]} \\
{[1.0,2.0]}\end{array}$ & $\begin{array}{l}{[6.0,8.0]} \\
{[8.0,15]}\end{array}$ & $\begin{array}{l}{[0.4,1.2]} \\
{[0.4-1.2]}\end{array}$ \\
\hline \multicolumn{10}{|c|}{ South-inner } \\
\hline $\begin{array}{l}\mathrm{A} \\
\mathrm{B}\end{array}$ & $\begin{array}{l}40 \\
35\end{array}$ & $\begin{array}{l}1.81 \\
1.59\end{array}$ & $\begin{array}{c}{[-7,11]} \\
{[-5,9]}\end{array}$ & $\begin{array}{l}{[0.5,1.3]} \\
{[0.5,1.2]}\end{array}$ & $\begin{array}{l}{[-0.2,-0.9]} \\
{[-0.3,-0.8]}\end{array}$ & $\begin{array}{l}{[0.9,3.5]} \\
{[0.9,1.2]}\end{array}$ & $\begin{array}{l}{[1.0,2.5]} \\
{[1.2,2.2]}\end{array}$ & $\begin{array}{l}{[8.5,15]} \\
{[3.5,5.5]}\end{array}$ & $\begin{array}{l}{[0.1,0.6]} \\
{[0.2,0.5]}\end{array}$ \\
\hline
\end{tabular}

Note. - A and B denote ASS and BSS models, correspondingly. The parameters $\alpha$ and $\beta$ determine the strengths of the disk and halo components as explained in Sect. 4.3

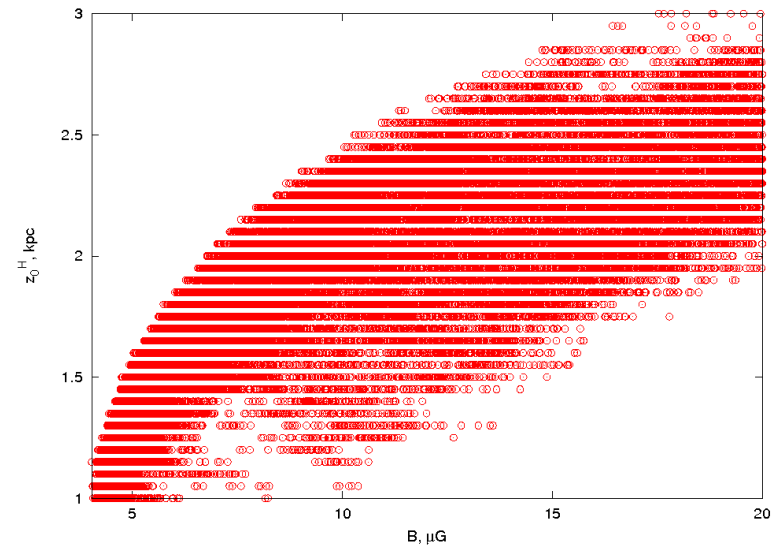

FIG. 12.- Height of the halo $z_{0}^{H}$ vs. its strength $B_{0}^{H}$. Each dot represents an acceptable model. The degeneracy could be easily seen: a very strong halo field gives an acceptable fit provided the vertical scale $z_{0}^{H}$ is high. 

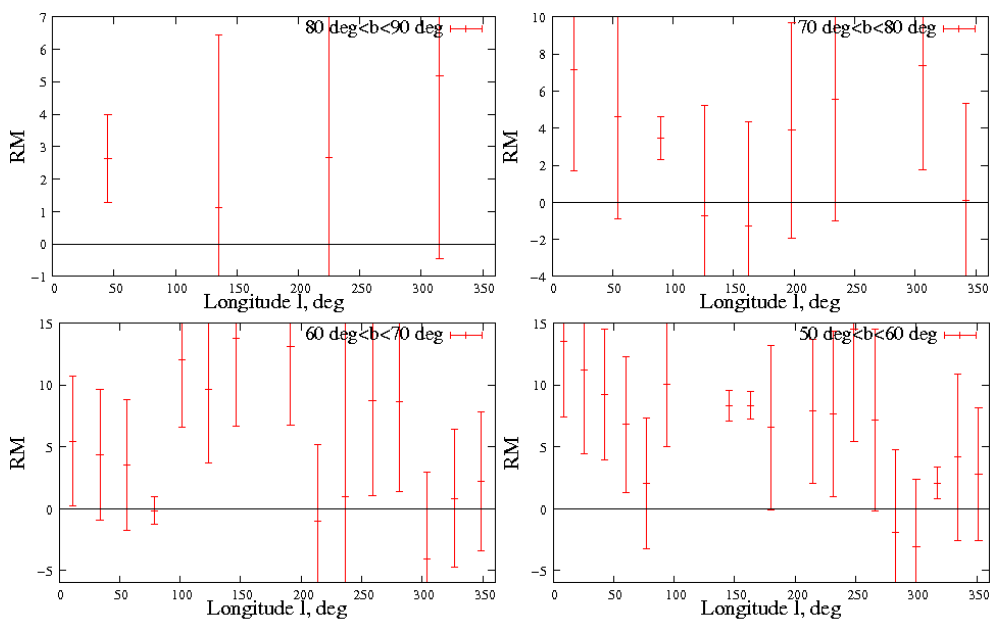

FIG. 13. - NVSS data for $b>50^{\circ}$. The bias towards positive values of RMs, perhaps due to the small vertical component $B_{z}$, could be seen.
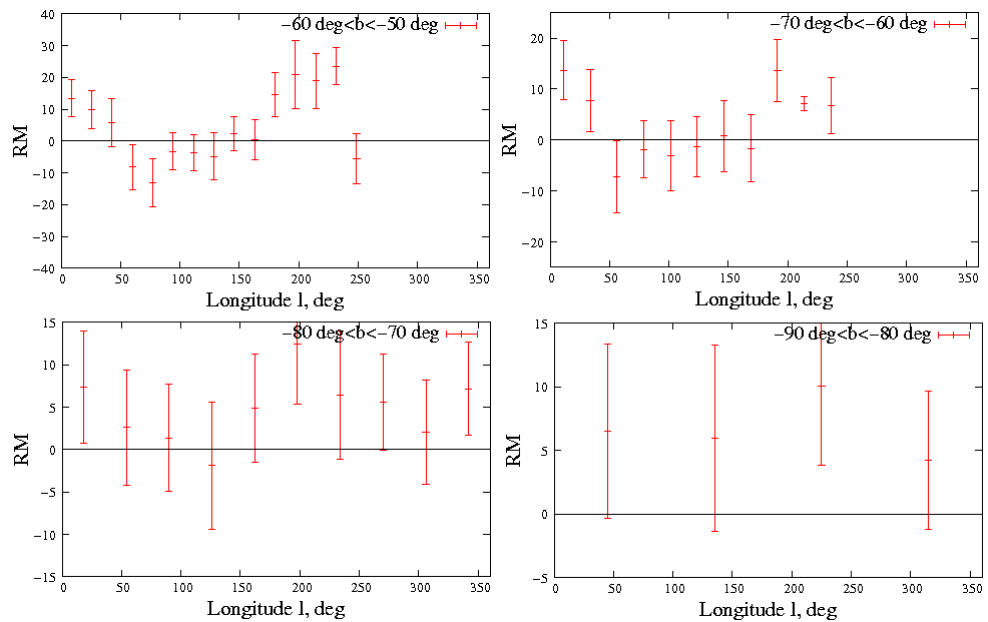

FIG. 14. - NVSS data for $b<-50^{\circ}$. Bias towards positive values of RMs could be seen here as well.

\section{REFERENCES}

Beck, R. 2001, Space Sci. Rev., 99, 243

Beck, R. 2008, in American Institute of Physics Conference Series, Vol. 1085, American Institute of Physics Conference Series, ed. F. A. Aharonian, W. Hofmann, \& F. Rieger, 83-96

Brandenburg, A., Donner, K. J., Moss, D., et al. 1992, A\&A, 259, 453

Braun, R., Heald, G., \& Beck, R. 2010, A\&A, 514, A42+

Brown, J. C., Haverkorn, M., Gaensler, B. M., et al. 2007, ApJ, 663,258

Brown, J. C., Taylor, A. R., \& Jackel, B. J. 2003, ApJS, 145, 213

Condon, J. J., Cotton, W. D., Greisen, E. W., et al. 1998, AJ, 115,1693

Cordes, J. M., \& Lazio, T. J. W. 2002, arXiv:astro-ph/0207156

Crutcher, R. M. 1999, ApJ, 520, 706

Frick, P., Stepanov, R., Shukurov, A., \& Sokoloff, D. 2001, MNRAS, 325, 649

Gaensler, B. M., Madsen, G. J., Chatterjee, S., \& Mao, S. A. 2008, Publ. Astr. Soc. Australia, 25, 184

Han, J. L., Manchester, R. N., Berkhuijsen, E. M., \& Beck, R. 1997, A\&A, 322, 98

Han, J. L., Manchester, R. N., Lyne, A. G., Qiao, G. J., \& van Straten, W. 2006, ApJ, 642, 868

Han, J. L., \& Qiao, G. J. 1994, A\&A, 288, 759

Haslam, C. G. T., Salter, C. J., Stoffel, H., \& Wilson, W. E. 1982, A\&AS, 47, 1
Heiles, C. 1996, ApJ, 642, 316

Jaffe, T. R., Leahy, J. P., Banday, A. J., et al. 2010, MNRAS, 401,1013

Jansson, R., Farrar, G. R., Waelkens, A. H., \& Enßlin, T. A. 2009, JCAP, 7, 21

Klein, U., Mack, K., Gregorini, L., \& Vigotti, M. 2003, A\&A, 406, 579

Krause, M. 2009, in Revista Mexicana de Astronomia y Astrofisica Conference Series, Vol. 36, Revista Mexicana de Astronomia y Astrofisica Conference Series, 25-29

Kronberg, P. P., \& Newton-McGee, K. J. 2011, Publ. Astr. Soc. Australia, 82, 171

Mao, S. A., Gaensler, B. M., Haverkorn, M., et al. 2010, ApJ, 714,1170

Mao, S. A., Gaensler, B. M., Stanimirović, S., et al. 2008, ApJ, 688,1029

Men, H., Ferrière, K., \& Han, J. L. 2008, A\&A, 486, 819

Nishiyama, S., et al. 2010, ApJ, 722, L23

Prouza, M., \& Šmída, R. 2003, A\&A, 410, 1

Ruiz-Granados, B., Rubiño-Martín, J. A., \& Battaner, E. 2010, A\&A, 522, A73+

Simard-Normandin, M., \& Kronberg, P. P. 1979, Nature, 279, 115

-. 1980, ApJ, 242, 74

Simard-Normandin, M., Kronberg, P. P., \& Button, S. 1981, ApJS, 45, 97 
Sofue, Y., \& Fujimoto, M. 1983, ApJ, 265, 722

Stanev, T. 1997, ApJ, 479, 290

Stil, J. M., Taylor, A. R., \& Sunstrum, C. 2011, ApJ, 726, 4

Sun, X., \& Reich, W. 2010, Research in Astronomy and Astrophysics, 10, 1287

Sun, X. H., Reich, W., Waelkens, A., \& Enßlin, T. A. 2008, A\&A, 477,573

Taylor, A. R., Stil, J. M., \& Sunstrum, C. 2009, ApJ, 702, 1230
Tinyakov, P. G., \& Tkachev, I. I. 2002, Astroparticle Physics, 18, 165

-. 2005, Astroparticle Physics, 24, 32

Vallée, J. P. 2005, ApJ, 619, 297

Wielebinski, R. 2005, in Lecture Notes in Physics, Berlin Springer Verlag, Vol. 664, Cosmic Magnetic Fields, ed. R. Wielebinski \& R. Beck, 89-+

Wolleben, M., et al. 2010, ApJ, 724, L48 